\title{
Estudo comparativo dos meios de contraste baritado e iodado-iônico e não-iônico no trato respiratório de ratos $^{1}$
}

\author{
Maria de Lourdes Pessole Biondo-Simões ${ }^{2}$ \\ Fernando Hintz Greca ${ }^{3}$ \\ Maurizzio Pedrazzani ${ }^{4}$ \\ Heraldo O. Mello Jr ${ }^{5}$ \\ Sérgio Ossamu Ioshii ${ }^{6}$ \\ Maria Francisca Kaizu Cavalcanti ${ }^{7}$ \\ Débora R. Voltarelli de Souza ${ }^{7}$ \\ Letícia Yurie Kimura ${ }^{8}$
}

Biondo-Simões MLP, Greca FH, Pedrazzani M, Mello Jr HO, Ioshii SO, Cavalcanti MFK, Souza DRV, Kimura LY. Estudo comparativo dos meios de contraste baritado e iodado-iônico e não-iônico no trato respiratório de ratos. Acta Cir Bras [serial online] 2003 Set-Out;18(5). Disponível em URL: http://www.scielo.br/acb.

RESUMO - Objetivo: Durante estudos radiológicos pode ocorrer a passagem do meio de contraste para a árvore traqueo-brônquica, determinando reações adversas e até fatais. O objetivo deste estudo é conhecer as alterações desencadeadas no trato respiratório pela presença do meio de contraste. Métodos: Utilizaram-se 91 ratos divididos, aleatoriamente, em 4 grupos. Um grupo de controle não manipulado com 7 animais e os demais grupos com 21 ratos. O grupo controle manipulado recebeu solução fisiológica e os grupos de experimento: bário, iodo ônico e iodo não iônico conforme o grupo a que pertenciam. Sob anestesia e visibilização direta da traquéia, injetouse $0,75 \mathrm{ml} / \mathrm{kg}(0,25 \mathrm{ml})$ do meio de contraste de acordo com o grupo ao qual pertencesse o animal. Realizaram-se as aferições com 1 hora, 1 dia e 1 semana. Os ratos foram sorteados para a autanásia nos três tempos estudados. Fez-se documentação radiográfica seguida da ressecção dos pulmões e exame histo-patológico. Resultados: Na primeira hora, no grupo que recebeu bário, os campos médios e o superior direito ficaram acometidos. Nos grupos que receberam iodo iônico e iodo não iônico, todo o pulmão direito mostrava-se opacificado. Nas avaliações de uma dia e 1 semana os grupos que receberam iodo estavam normais enquanto que o grupo que recebeu bário mostrava comprometimento dos campos médios. No estudo histo-patológico observou-se, no grupo que recebeu bário, na avaliação de uma semana, grande quantidade de macrófagos intra-alveolares, perivasculares e peribrônquicos. Conclusão: Os contrastes iodados iônico e não iônico levam a alterações passageiras enquanto que o bário promove reação inflamatória crônica com manutenção da tradução radiográfica, no rato.

DESCRITORES - Bário. Meios de contraste. Iodo. Pulmão.

1. Trabalho desenvolvido na Disciplina de Técnica Operatória e Cirurgia Experimental da Pontifícia Universidade Católica do Paraná (PUC-PR) e Serviço de Radiologia da Santa Casa de Misericórdia (SCM) de Curitiba.

2. Professora Adjunta da Disciplina de Técnica Operatória e Cirurgia Experimental da PUC-PR. Doutora em Cirurgia Experimental pela Escola Paulista de Medicina. Membro da SBCP e da SOBRADPEC. Titular do CBC-PR.

3. Professor Coordenador da Disciplina de Técnica Operatória e Cirurgia Experimental da PUC-PR. Doutor em Cirurgia Experimental pela Escola Paulista de Medicina. Membro da SBCP e da SOBRADPEC.

4. Professor Adjunto da Disciplina de Radiologia da PUC-PR.

5. Professor Coordenador da Disciplina de Radiologia da PUC-PR.

6. Professor Adjunto da Disciplina de Patologia da PUC-PR.

7. Médicas Residentes do Serviço de Radiologia da Santa Casa de Misericórdia - PUC-PR

8. Acadêmica de Medicina da PUC-PR. 


\section{Introdução}

Durante o estudo contrastado do trato digestivo alto para os exames do refluxo gastro-esofágico, de fístulas traqueo-esofagianas, de distúrbios da deglutição e de outras patologias, pode ocorrer a passagem do meio de contraste para a árvore traqueo-brônquioalveolar, determinando reações adversas e até mesmo fatais $^{3,4}$.

Há controvérsias quanto ao uso do contraste ideal e quanto à falta de padronização para a realização dos exames. Alguns autores defendem o uso do bário, enquanto que outros preferem o contraste iodado, não havendo uma definição apropriada na literatura quanto ao meio de contraste mais eficaz e com menos efeitos adversos.Um grande número de riscos e de reações são descritas, variando desde alterações inflamatórias, granuloma de corpo estranho, bronquite aguda ou crônica, broncopneumonia, atelectasia, edema, enfisema, necrose, fibrose, podendo até levar à morte. Fung e col. (1990) relataram aspiração do meio de contraste, durante a investigação do trato respiratório superior e a literatura refere que este acidente pode ocorrer em até $8 \%$ dos exames ${ }^{4-13}$.

Os meios de contraste que chegariam perto do ideal seriam os do tipo iso-osmolar, não iônico ${ }^{5,14}$. No entanto estes não são disponíveis no nosso país, devido ao alto custo.

O objetivo do presente estudo é comparar os efeitos dos diferentes meios de contraste utilizados em nosso país e avaliar seus riscos para o trato respiratório, por meio de um estudo experimental em ratos.

\section{Métodos}

Este estudo obedeceu as normas de pesquisa com animais preconizadas pelo Colégio Brasileiro de Experimentação Animal (COBEA), instituição filiada ao International Council for Laboratory Animal Science e à Lei Federal 6.638.

Utilizaram-se no experimento 84 ratos machos, adultos jovens (Rattus norvegicus albinus, Rodentia mammalia), Wistar-TECPAR, procedentes do biotério do Instituto Tecnológico do Paraná.

Distribuíram-se os animais, aleatoriamente, em 5 grupos. Um desses grupos recebeu 7 animais e constituiu o controle não manipulado. Os demais grupos receberam 21 ratos e eram assim chamados: grupo controle manipulado que recebeu solução fisiológica (SF), grupo experimental bário (B), grupo experimental iodo iônico (II) e grupo experimental iodo não iônico (INI).
Sob anestesia, em cada um dos ratos dos grupos SF, B, II e INI procedeu-se à depilação e à anti-sepsia da região cervical anterior. Fez-se uma incisão mediana de 1,5 cm interessando a pele, a tela subcutânea e o platisma. Identificou-se a rafe mediana, que aberta permitiu o afastamento da musculatura e exposição da traquéia.

Puncionou-se a traquéia com agulha de 14,5 x 3 acoplada à seringa de $1 \mathrm{ml}$ e injetou-se $0,25 \mathrm{ml}(0,75$ $\mathrm{ml} / \mathrm{kg}$ ) do meio de contraste estudado em cada grupo.

Em seguida realizou-se a cervicorrafia, com um único plano de síntese utilizando-se chuleio contínuo de fio monofilamentar de náilon 4.0.

Realizaram-se as avaliações após 1 hora, 1 dia e 1 semana, sorteando-se 7 animais de cada grupo para eutanásia, para cada um dos tempos. Desta forma cada grupo forneceu 3 subgrupos.

Faziam-se as radiografias de tórax dos ratos, com pulmões insuflados. A avaliação radiológica consistiu na observação da presença de áreas de hipotransparência ou opacificação, tendo-se dividido os campos pleuropulmonares em terços: basal, médio e superior, direito e esquerdo.

Após realizava-se uma incisão torácica mediana de aproximadamente $3 \mathrm{~cm}$, seguida de desarticulação do esterno e secção do diafragma bilateralmente. Dissecavam-se coração e pulmões cuidadosamente.

Utilizando-se uma seringa tipo insulina de $1 \mathrm{ml}$, ocluiu-se a traquéia com nó feito com fio de algodão 2-0 e expandiam-se os pulmões com formalina a $10 \%$ tamponada. Mergulharam-se os pulmões expandidos em frascos que continham formalina tamponada a $10 \%$. Após emblocagem em parafina obtiveram-se cortes com 4 micrômetros de espessura que, corados pela hematoxilina-eosina, permitiram avaliar o processo inflamatório.

Avaliou-se o processo inflamatório levando-se em conta o predomínio dos tipos celulares:

1. reação inflamatória aguda (espessamento intersticial alveolar e bronquilar, hiperplasia do epitélio e presença de neutrófilos); e

2. reação inflamatória crônica (presença de macrófagos intra-alveolares, peri-brônquicos e perivasculares e pneumócitos hiperplásicos).

Classificou-se a reação inflamatória em graus: 0 = ausente; 1 = discreta; $2=$ moderada e $3=$ intensa.

Submeteram-se os resultados à tratamento estatístico, pelos testes do qui-quadrado e exato de Fisher. Estabeleceu-se $\mathrm{p}=0,05$ como nível para rejeição da hipótese de nulidade. 


\section{Resultados}

Todos os animais toleraram bem a anestesia e o procedimento cirúrgico.

$\mathrm{Na}$ avaliação de 1 hora, nas radiografias dos ratos que receberam sulfato de bário, encontrou-se opacificação dos terços médios e superiores direitos em $100 \%$ delas e do terço basal direito em $71 \%$. Viu-se opacificação dos terços médios esquerdos em $71 \%$ dos exames.
Nas radiografias dos animais, aos quais se administrou iodo iônico, encontrou-se hipotransparência em terço médio direito $(71 \%)$ e nos terços basal e superior direitos (54\%). Nos campos esquerdos estavam hipotransparentes $43 \%$ dos campos médios e $28 \%$ dos campos basais (Figura 1A).

Os exames dos animais que receberam iodo não iônico as hipotransparências estavam localizadas no terço médio direito em $71 \%$ deles e no terço basal em $57 \%$ (Figura 1B).

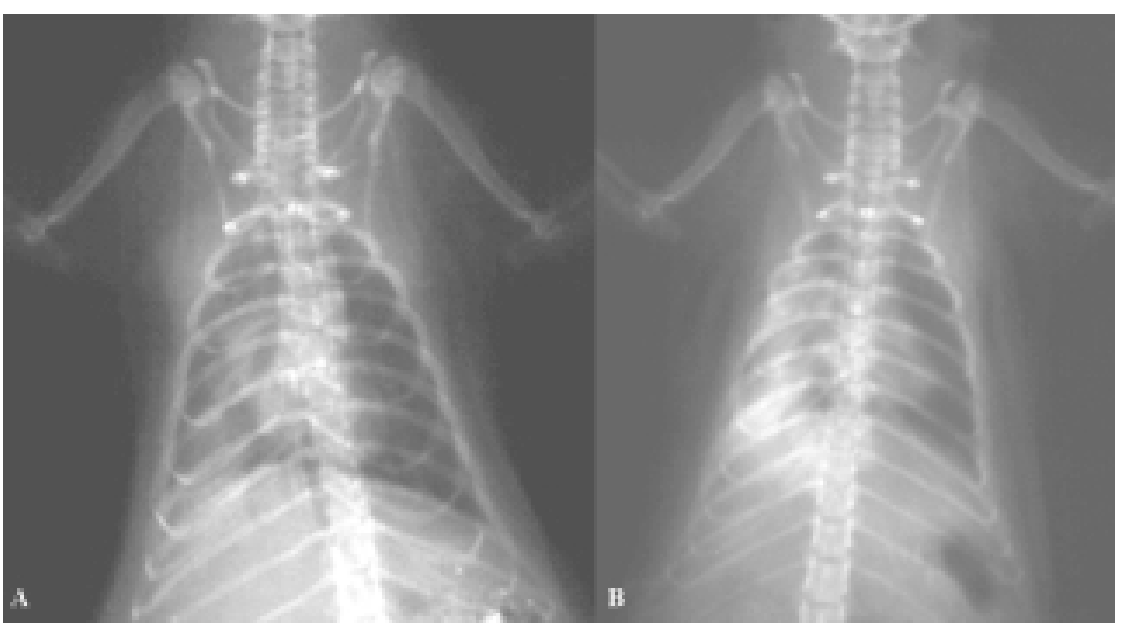

FIGURE 1 - Radiografias dos animais do grupo iodo iônico (A) e iodo não iônico (B), na avaliação de 1 hora mostrando hipotransparência nos terços basal e medial direitos.

Se considerarmos apenas a positividade do exame verifica-se que não há diferença significante entre os grupos que receberam meios de contraste $(p=0,2308)$ e embora exista diferença uma predominância pelo pulmão direito, esta também não é significante. As áreas pulmonares comprometidas, neste tempo, foram os terços basais e médios (tabela 1). Como cada radiografia forneceu 6 áreas, 3 à direita e 3 à esquerda, pode-se perceber que com 1 hora não existe diferença importante entre os exames dos ratos que receberam bário e os dos que receberam iodo iônico $(\mathrm{p}=0,2924)$, porém existe importante diferença entre eles e os dos que receberam iodo não iônico $(\mathrm{p}=0,0110)$ (Tabela 2).

TABELA 1 - Áreas pulmonares comprometidas considerando os meios de contrastes empregados na avaliação de 1 hora (considere-se que cada radiografia forneceu seis áreas).

\begin{tabular}{ccccc}
\hline \multicolumn{5}{c}{ áreas pulmonares comprometidas } \\
grupo & basal & medio & superior & TOTAL \\
\hline B & 10 & 7 & 0 & 17 \\
II & 6 & 8 & 0 & 14 \\
INI & 4 & 5 & 0 & 9 \\
Total & 20 & 20 & 0 & 40 \\
\hline
\end{tabular}

$\mathrm{B}=$ bário, $\mathrm{II}=$ iodo iônico, $\mathrm{INI}=$ iodo não iônico

Teste exato de Fisher

B x II $p=0,3760$

B x INI $\mathrm{p}=0,3867$

II $x$ INI $p=0,6369$ 
TABELA 2 - Número de áreas pulmonares comprometidas na avaliação 1 hora.

\begin{tabular}{cccccc}
\hline \multicolumn{7}{c}{ áreas pulmonares } \\
grupo & comprometidas & \multicolumn{4}{c}{ não comprometidas } \\
\hline B & número & $\%$ & número & $\%$ & TOTAL \\
II & 17 & 80,95 & 4 & 19,05 & 21 \\
INI & 14 & 66,67 & 7 & 33,33 & 21 \\
Total & 9 & 42,86 & 12 & 57,14 & 21 \\
& 40 & 63,49 & 23 & 36,51 & 63 \\
\hline
\end{tabular}

$\mathrm{B}=$ bário, II = iodo iônico, INI = iodo não iônico

Teste exato de Fisher

B x II $p=0,2924$

B x INI $\mathrm{p}=0,0110^{*}$

II $x$ INI $p=0,1211$

Na avaliação de 1 dia, os exames do grupo que recebeu bário demonstraram opacificação dos terços médios e superior direito em $57 \%$ dos exames e do terço basal em $43 \%$. O terço basal esquerdo apareceu comprometido em $28 \%$ dos exames. Não se encontraram alterações nos exames radiológicos dos animais que receberam iodo iônico e não iônico.

Na avaliação de 1 semana apresentava, ao exame radilógico, opacificação do terço médio direito em $100 \%$ dos animais, do terço superior em $86 \%$ e do terço basal em $71 \%$. Os terços basais e apicais esquerdos estavam comprometidos em $43 \%$ e $28 \%$ respectivamente (Figura 2). Os exames dos animais que receberam iodo iônico estavam normais e $28 \%$ dos que receberam iodo Não iônico tinham leve comprometimento do terço superior direito.

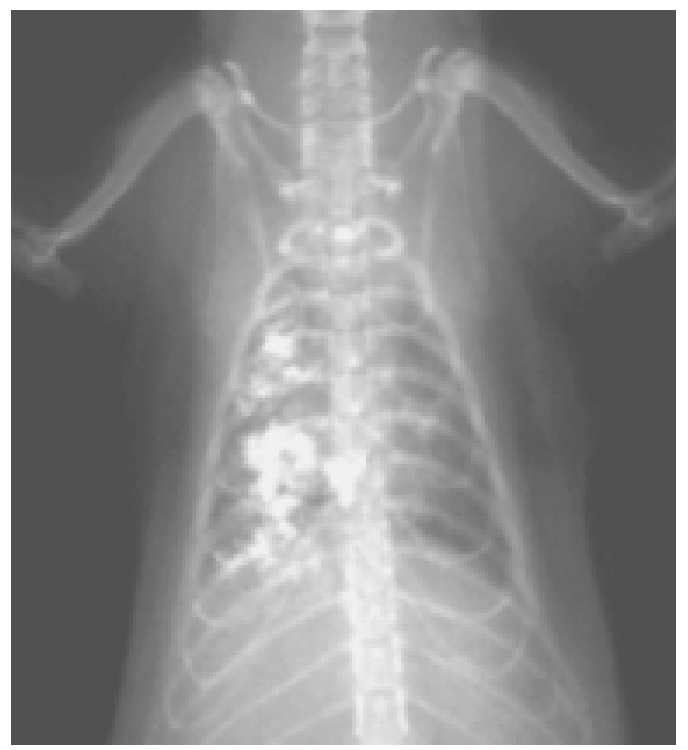

FIGURE 2 - Radiografia de animal do grupo bário na avaliação de 1 semana mostrando áreas de opacificação no pulmão direito, nos terços superior, médio e basal.
Os exames do grupo controle não manipulado e dos que receberam solução fisiológica estavam todos normais. No grupo do B 7 dias as regiões dos pulmões mais acometidas foram os campos médios (100\%), seguido do lobo superior direito $(86 \%)$. Já nos outros grupos não houve alterações relevantes.

A avaliação histológica revelou, no grupo controle e no grupo que recebeu solução fisiológica raros macrófagos intra-alveolares, perivasculares e peribronquiais nos 3 tempos estudados.

Nos grupos que receberam iodo iônico e não iônico ocorreu aumento progressivo do número de macrófagos intra-alveolares, perivasculares e peribronquiais.

Nos cortes histológicos dos pulmões, do grupo que recebeu sulfato de bário, nos exames de 1 hora e de 1 dia, observava-se maior número de macrófagos e era possível dizer que os cortes do pulmão direito os apresentavam em maior número que o esquerdo. Nos exames de 1 exame viu-se aumento importante do número de macrófagos associado com hiperplasia dos pneumócitos o que traduz importante reação inflamatória crônica.

A análise do número de macrófagos intra-alveolares de todos os grupos, é estatisticamente significante quando se comparam os cortes dos pulmões controle e dos que receberam solução fisiológica com os dos que receberam bário com 1 dia e 1 semana ( 1 dia $\mathrm{p}=0,0023$ e 1 semana $\mathrm{p}=0,0029$ ). O mesmo acontece quando se comparam com os encontrados nos cortes dos pulmões dos ratos que receberam iodo iônico com 1 semana $(\mathrm{p}=0,0349)$. A comparação entre os grupos bário e iodo iônico e não iônico, na avaliação de uma semana demonstrou elevada concentração de macrófagos peribronquicos no grupo bário $(\mathrm{p}=0,0002)$. A análise dos macrófagos peri-vasculares, com 1 dia, demonstrou-os em maior número nos exames dos animais que receberam bário quando comparados com 
os dos que receberam solução fisiológica ( $\mathrm{p}=0,0349)$. Isso se repetiu na comparação com os encontrados nos exames dos que receberam bário e iodo não iônico ( $\mathrm{p}=0,0030)$. A comparação entre os grupos iodo iônico e não iônico, mostrou-os, em maior número no grupo iodo iônico $(\mathrm{p}=0,0104)$. Na avaliação com 1 semana foram vistos em maior número no grupo bário quando comparado ao grupo iodo iônico $(\mathrm{p}=0,0002)$ e iodo não iônico $(\mathrm{p}=0,0003)$. Neste tempo encontraram-se pneumócitos hiperplásicos em grande número, no grupo bário ( $\mathrm{p}=0,0023$ comparado com o grupo iodo iônico e $\mathrm{p}=0,0105$ quando comparado ao grupo iodo não iônico). Comparados os grupos iodo não se verificou diferença $(\mathrm{p}=0,0500)$.

A Figura 3 mostra aspectos histológicos dos pulmões dos 4 grupos.

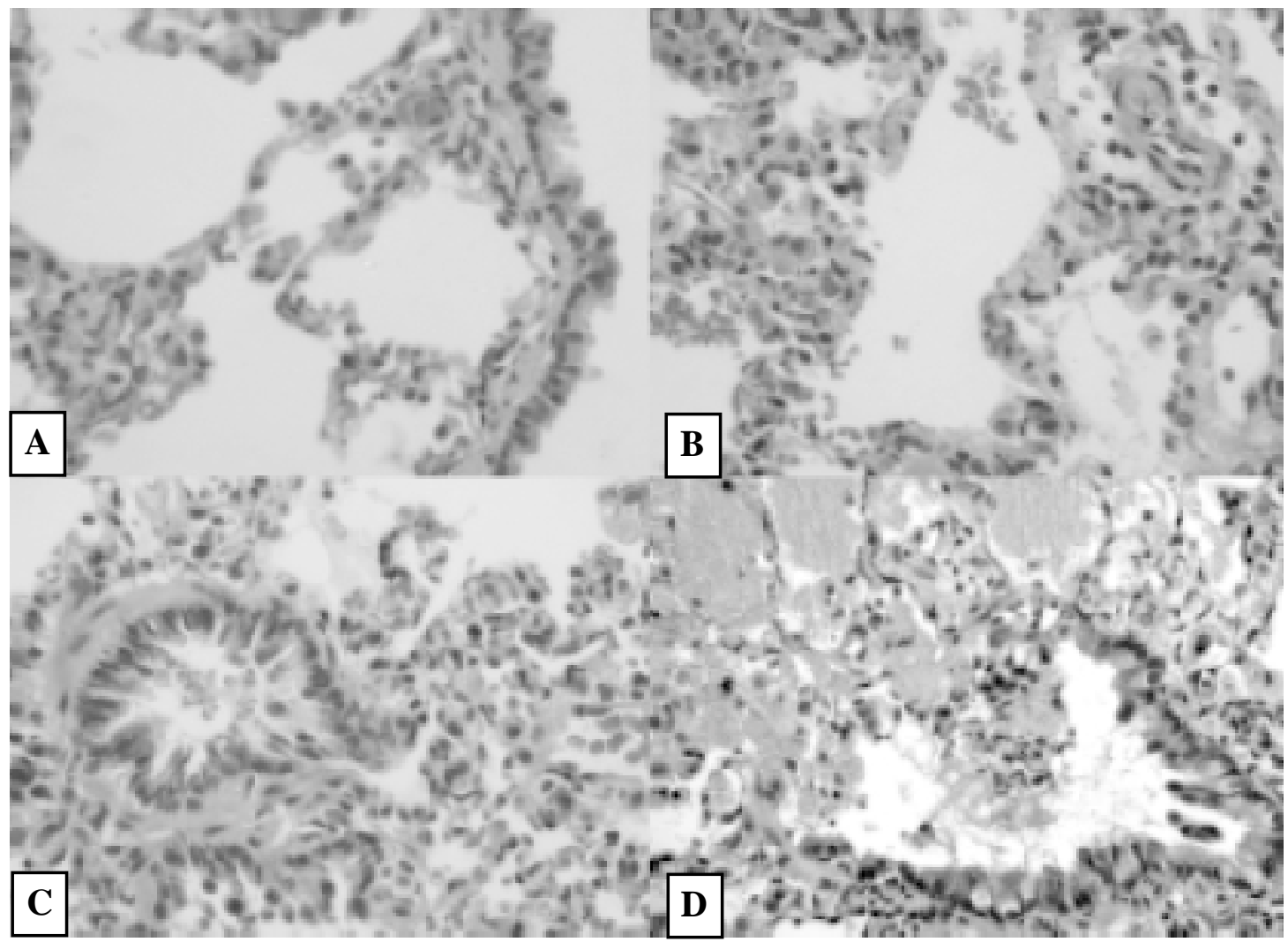

FIGURE 3 - Fotomicrografia de cortes histológicos de pulmão direito na avaliação de 1 semana (HE - 400 X)

A) grupo II, espaços alveolares com discreto aumento do número de macrófagos;

B) grupo INI, distribuição intra-alveolar e perivascular dos macrófagos e edema;

C) grupo B, bronquio terminal com hiperplasia celular e macrófagos intralveolares;

D) grupo $\mathrm{B}$, bronquio terminal parcialmente lesado, com proliferação de macrófagos associada a infiltrado de neutrófilos e monomorfonucleares.

\section{Discussão}

Existem poucos estudos na literatura avaliando alterações radiológicas e histológicas pulmonares, causadas pelos meios de contraste.

Os tempos de avaliação: 1 hora, 1dia e 1 semana foram selecionados de acordo com a literatura ${ }^{3,5,8}$. McAlister e Askin (1983) relataram maior fracionamento do tempo de avaliação, que variava entre 15 minutos e 9 mêses ${ }^{3}$.

Os achados radiográficos não foram expressivos nos grupos tratados com iodo nas avaliações de 1 dia e
1 semana. Ginai e col. (1993) e Thompson e col. (1997) relataram resultados semelhantes, ao passo que French e col. (1970), observaram mais opacificação na série tratada com iodo iônico ${ }^{5,8,9}$

Neste estudo pode-se verificar que a extensão da área pulmonar comprometida foi maior no grupo tratado com bário quando comparado ao iodo não iônico na avaliação de 1 hora $(\mathrm{p}=0,0110)$ e nas avaliações de 1 semana apenas o grupo tratado com bário apresentava alterações radiológicas. Estes dados são semelhantes aos descritos na literatura ${ }^{3,9}$. 
Fibrose não foi avaliada neste estudo porque o tempo usado para avaliação não era suficiente para que ela se instalasse. O numero de macrófagos foi exuberante no grupo que recebeu bário. Achado este já descrito por McAlister e Askin $(1983)^{3}$ e Frech e col. $(1970)^{8}$.

A reação inflamatória, neste estudo, foi significantemente maior no grupo que recebeu bário, reação esta que persistiu até a avalição de 1 semana quando, inclusive, apareceram pneumócitos hiperplásicos. Os grupos tratados com iodo iônico ou não mostraram reação inflamatória mínima e não significante neste tempo. Ginai e col. (1993) demonstraram resultados semelhantes ${ }^{14}$.

O bário é mais viscoso e distribui-se menos amplamente, com tendência a se localizar ${ }^{8}$. Contudo neste estudo o comprometimento pulmonar foi mais difuso do que com os meios de contraste iodados, não obstante, ter-se concentrado no terço médio.

Como no estudo de Ginai e col.(1993), não se encontrou neste experimento derrame pleural, pneumotórax ou colapso pulmonar5.

Embora sejam relatadas mortes por aspiração, isto não aconteceu neste estudo.

Muitos autores ${ }^{5,14}$ determinaram que o melhor meio de contraste seria o do tipo iso-osmolar nãoiônico ${ }^{3,5,8,12,16}$. No entanto, o alto custo, o torna não está disponível em nosso meio.

Assim analisando os resultados deste estudo parece possível admitir que o sulfato de bário promove maior comprometimento pulmonar traduzido por opacificação dos campos pulmonares e intensa reação inflamatória crônica.

\section{Conclusão}

No rato, os contrastes iodados iônico e não iônico levam a alterações pulmonares passageiras enquanto que o bário promove reação inflamatória crônica com manutenção da tradução radiográfica.

\section{Referências}

1. Gombar KK, Singh B, Chhbra B. Fatal pulmonary aspiration of barium during oesophagography. Trop Doct 1995; 25:184-5.

2. Tsokos M, Schulz F, Vogel H. Barium aspiration with fatal outcome. Aktuelle Radiol 1998; 8:201-3.

3. McAlister WH, Askin FB. The effect of some contrast agents in the lung: an experimental study in the rat and dog. AJR $1983 ; 140: 245-51$

4. Fung KP; Seagram G; Pasieka, J; Trevenen C; Machida H; Scott B. Investigation and outcome of 121 infants and children requiruing Nissen fundoplication for the management of gastroesophageal reflix. Clin Invest Med 1990: 13: 237-46.

5. Ginai AZ, Bubberman A, Zondervan PE, Leeuwn WV, Morcos SK. The histological response of the lungs of rats to potentially suitable water soluble bronchographic contrast agents iotrolan (a non-ionic dimmer) and iopamidol (a non-ionic monomer). Br J Radio 1993; 66:773-7.

6. Teasell RW, McRae M, Heitzner J, Bhardwaj A, Finestone H. frequency of videofluoroscopic modified barium swallow studies and pneumonia in stroke rehabilitation patients: a comparative study. Arch Phys Med Reabil 1999; 80:294-8.

7. Buecker A, Wein BB, Neuerburg JM, Guenther RW. Esophageal perforation: comparison of use of aqueous and bariumcontaining contrast media. Radiology 1997; 202:683-6.

8. Frech RS, Davie JM, Adatepe M, Feldhaus R, McAlister H. comparison of barium sulfate and oral $40 \%$ diatrizoate injected into the trachea of dogs. Radiology 1970; 95:299-303.

9. Thompson IM, Whittlesey GC, Slovis TL, Chang CH, Cullen ML, Philippart AI, Stockmann PS, Adkins ES, Klein MD Evaluation of contrast media for bronchography. Pediatr Radiol 1997; 27:598-605.

10. Buschman DL. Barium sulfate bronchography: report of a complication. Chest 1991; 99:747-9.

11. Trulzsch DV, Penmetsa A, Karim A, Evans DA. Gastrografininduced aspiration pneumonia: a lethal complication of computed tomography. Southern Med J 1992; 85(12):1255-6.

12. Pracy JPM, Montgomery PQ. Acute pneumonitis caused by low-density barium sulphate aspiration. J Laryngol Otol 1993; 107:347-348.

13. Ansell G. A national survey of radiological complications: interim report. Clin Radiol 1968; 19:175-91.

14. Ginai AZ. Experimental evaluation of various available contrast agents for use in the gastrointestinal tract in case of suspected leakage: effects on pleura. Br J Radiol 1986; 59:887-94.

15. Ratcliffe, JF. Effect of contrast agents on the lungs. Br J Radiol 1985; 58:574-6.

16. Brick SH, Caroline DF, Lev-Toaff AS, Friedman AC, Grumbach K, Radecki PD. Esophageal disruption: evaluiation with iohexol esophagography. Radiology 1988; 169:141-3. 
Biondo-Simões MLP, Greca FH, Pedrazzani M, Mello Jr HO, Ioshii SO, Cavalcanti MFK, Souza DRV, Kimura LY. Comparative study of barium and iodine agents - ionic and nonionic on the respiratory tract of the rats. Acta Cir Bras [serial online] 2003 Sept-Oct;18(5). Available from URL: http://www.scielo.br/acb.

ABSTRACT - Purpose: During roadiologic studies, passage of the contrast medium to the tracheobronchial tree may occur, causibng adverse or even fatal reactions. The objective of the present study was to determine the changes in the respiratory tract triggered by the presence of contrast medium. Methods: Ninety-one rats were divided at random into 5 groups, i.e., an intact control group of 7 animals and 4 groups of 21 rats each. The manipulated control group received physiological saline and the experimental groups respectively received barium, ionic iodine and non-ionic iodine. Under anesthesia and direct visualization of the trachea, $0.75 \mathrm{ml} / \mathrm{kg}(0.25 \mathrm{ml})$ of the contrast medium was injected into the animals of each group. The effects of the procedure were determined after 1 hour, 1 day and 1 week, with the animals being sacrificed at each time point by drawing lots. Radiographic documentation was obtained, followed by resection and histopathologic examination of the lungs. Results: After 1 hour, the middle fields and the right upper field were involved in the group injected with barium. In the groups injected with ionic iodine and non-ionic iodine, the entire right lung was found to be opacified. After 1 day and 1 week, the groups injected with iodine were normal, whereas the group injected with barium showed involvement of the middle fields. After 1 week, histopathologic examination revealed that the group injected with barium exhibited large amounts of intra-alveolar, perivascular and peribronchial macrophages Conclusion: The ionic and non-ionic iodine contrast media led to temporary changes, whereas barium promoted a chronic inflammatory reaction with maintenance of radiographic translation.

KEY WORDS - Barium. Contrast mediums. Iodine. Lung.

Conflito de interesse: nenhum

Fonte de financiamento: nenhuma

Correspondência:

Maria de Lourdes Pessole Biondo-Simões

Rua Ari José do Valle, 1987

82030-000 Curitiba - PR

Tel: (41)297-4359

biondo@avalon.sul.com.br

Data do recebimento: $15 / 06 / 2003$

Data da revisão: 25/06/2003

Data da aprovação: 05/07/2003 